\title{
Peierls transition as a spatially inhomogeneous gap suppression
}

\author{
V. Ya. Pokrovskii, A.V. Golovnya, and S.V. Zaitsev-Zotov \\ Institute of Radioengineering and Electronics, Russian Academy of Sciences, 103907 Moscow, Russia
}

(October 24, 2018)

\begin{abstract}
We propose a model of the Peierls transition (PT) taking into account amplitude fluctuations of the charge-density waves and spontaneous thermally activated suppression of the Peierls gap, akin to the phase slip process. The activation results in the exponential growth of the normal phase with increasing temperature. The model fairly describes the behavior of resistance, thermal expansion, Young modulus and specific heat both below and above the PT temperature $T_{P}$. The PT appears to have a unique nature: it does not comprise $T_{P}$ as a parameter, and at the same time it has features of the 1st order transition. The possible basis for the model is activation of non-interacting amplitude solitons perturbing large volumes around them.
\end{abstract}

PACS Numbers: 71.45.Lr, 71.30.+h, 68.35.Rh

Description of the Peierls transition (PT) in quasi 1dimensional (1D) conductors still remains a controversial problem. The widely used mean-field (MF) approach works poorly, firstly because of strong 1D fluctuations. E.g., it predicts the $\mathrm{PT}$ temperature $T_{P}$ much above the observed value. Say, for the typical compound $\mathrm{TaS}_{3}$, the energy gap $2 \Delta$ is $1600 \mathrm{~K}$, and the MF value of $T_{P}$ should be $2 \Delta / 3.5=460 \mathrm{~K}$, while the experimental value is $220 \mathrm{~K}$. The large fluctuations reveal themselves well above $T_{P}$ : the value of the pseudogap is close to the lowtemperature value $[1,2]$, the threshold non-linear conduction [3] indicates the charge-density wave (CDW) state within the fluctuating volumes. At present only qualitative attempts to explain these experimental facts are undertaken. Though certain success is achieved in fitting the behavior of different values near $T_{P}[4-6]$, the relations involved are semi-empirical, and their physical sense is not quite clear. Another treatment of the PT is given by the generalized Ehrenfest relation [7] between the specific heat, expansivity, Young modulus anomalies and stress-induced shift of the transition temperature. This relation works nice for some materials, e.g. for $\mathrm{K}_{0.3} \mathrm{MoO}_{3}$ - the blue bronze [4], but fails for others, like for $\mathrm{TaS}_{3}[8]$.

The general approach to the CDWs is to consider them as a spatially homogeneous state up to $T_{P}$. However, a recent theoretical study has demonstrated that thermal fluctuations of the CDW stress may be very large at $T \approx T_{P}$, so that the r.m.s. shift of the chemical potential level from the middle-gap position appears comparable with $\Delta[9]$, and one can expect temporal local suppression of the gap. So, it is natural to suppose that the Peierls state in the vicinity of $T_{P}$ should be considered as a mixture of the Peierls phase (the CDW) and the state with suppressed gap. Studies of noise have revealed spontaneous phase-slippage (PS) process $[10,11]$ in the vicinity of $T_{P}$, which also implies local temporal suppression of the Peierl gap. The PS is fairly described as a thermally activated process [12-14], so it would be intriguing to extend the aproach for the description of the PT.

In the present Letter we propose a model in which the fluctuations of $\Delta$ are phenomenologically introduced as thermally activated local gap suppression (LGS). With increasing $T$ this process gives rise to the activation growth of concentration of normal phase as $\exp (-W / T)$, $W \gg \Delta$. The approach can be extrapolated above the transition temperature. PT appears to be smeared out, but at the same time has features of the 1st order transition. The model appears to describe fairly the behavior of various parameters near $T_{P}$. The plausible basics for the model is the excitation of amplitude solitons perturbing considerable volumes around them.

To introduce the fluctuations, we shall use the following relation giving the frequency of the LGS acts per unit volume (see also [14]):

$$
f=f_{a} \exp (-W / T),
$$

where $f_{a}$ is an attempt frequency. Here the essential point is that each LGS act results in a temporal nucleation of the normal-state volume $v_{0}$ having certain lifetime $\tau$. Then the fraction of the normal-state volume due to the spontaneous LGS process is

$$
v=v_{0} \tau f
$$

This fraction grows exponentially with increasing $T$. At high enough temperature $v$ becomes of the order of 1 , so we should take into account the shrinking of the Peierlsstate volume. So, instead of (2) we obtain: $v=v_{0} \tau f(1-$ $v$ ), or

$$
v=\frac{v_{0} \tau f}{1+v_{0} \tau f} .
$$

This relation, the principal one for our model, gives growth of $v$ from 0 to 1 with increasing $T$. The growth has the form of a step centered at $T=W / \ln \left(v_{0} \tau f_{a}\right)$, at which $v=1 / 2$. The step is smeared out by $\approx T_{P}^{2} / W$ 


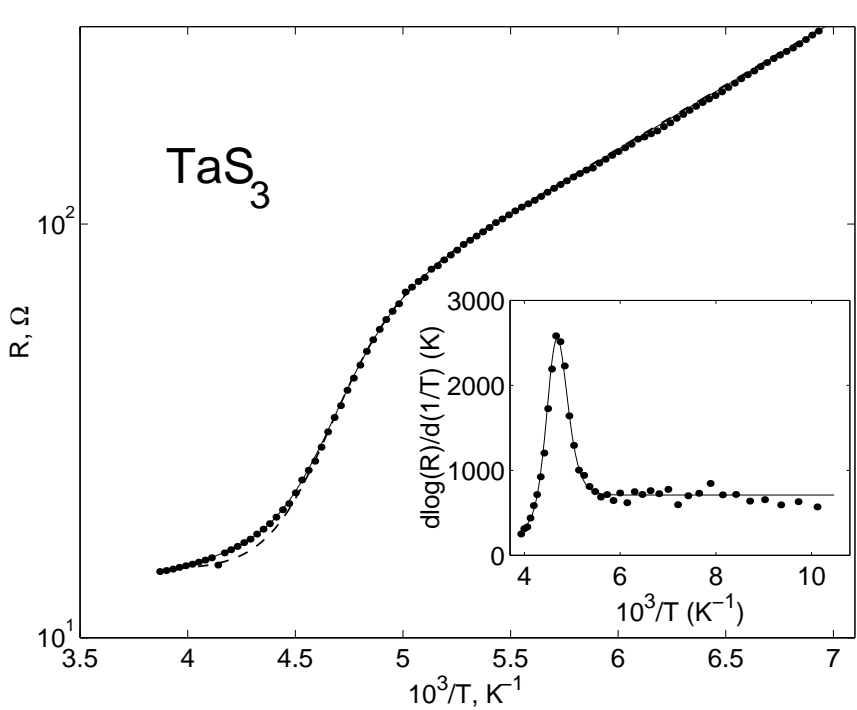

FIG. 1. Fits of a typical $R(T)$ curve for $\mathrm{TaS}_{3}$ (points) with Eq. (4); for the solid line $W=6600 \mathrm{~K}, v_{0} \tau f_{a}=3 \cdot 10^{13}$, $\Delta=710 \mathrm{~K}$. The broken line shows a similar fit with $B=0$. The inset shows the corresponding logarithmic derivatives.

(or $1 / W$ in the $1 / T$-scale). Extrapolation of the LGS description above $T_{P}$ gives a way to treat the entire PT. Within this approach the transition consists in the LGSinduced destruction of the CDW state.

Let us compare the resulting relation (3) with the experiment. We shall refer mostly to $\mathrm{TaS}_{3},-$ a typical CDW compound, which is among the widely studied quasi 1D conductors [15]. The large ratio $2 \Delta / T_{P}$ for $\mathrm{TaS}_{3}$, as well as highly anisotropic structure (the anisotropy of conductivity is about 100 at room temperature) argue for the strong fluctuations of the CDW order parameter.

We are beginning with the temperature dependence of resistance, the most common curve characterizing the PT. Within the present model we should calculate the resistance of a mixture of two phases with different resistivities, $\rho_{c}$ and $\rho_{n}$. We shall consider the Peierls-state resistivity $\rho_{c} \propto \exp (\Delta / T)$, and the normal state resistivity $\rho_{n}=A+B T$, where $A$ and $B$ are constants. To calculate the resulting resistivity one should consider a complex electric connection of the domains of each phase. For simplicity, we take the contribution of each phase to the resistivity to be just proportional to the volume fraction of the phase, as if for connection in series [16]:

$$
\rho=v \rho_{n}+(1-v) \rho_{c} .
$$

Note, that the fluctations are known to contribute to the conductivity of the Peierls phase due to thermal depinning of the CDW $[14,17,18]$. According to the model [14] this contribution is governed by the LGS as well and grows as $\exp (-W / T)$. Here we shall not distinguish it from that of the normal phase.

Fig. 1 presents an example of a fit of the $R(T)$ curve for $\mathrm{TaS}_{3}$ with Eq. (4). The fitting is splendid, but one should

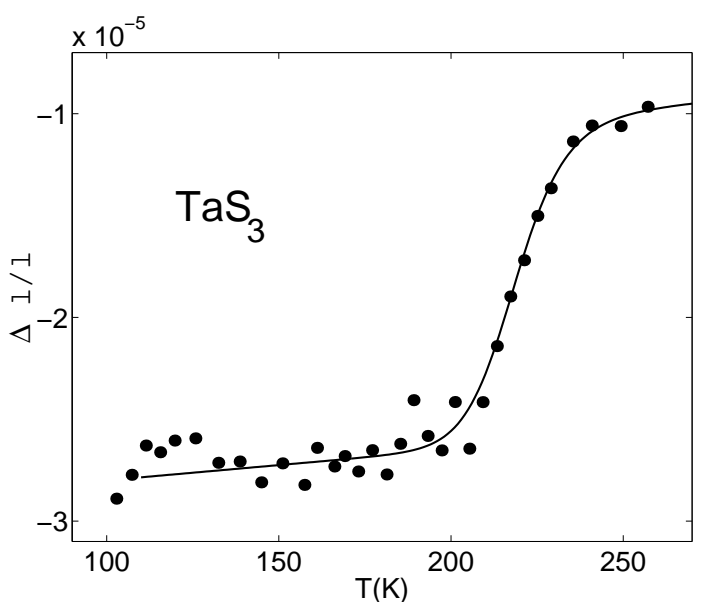

FIG. $2 . \quad \delta l / l$ vs. $T$ for a $\mathrm{TaS}_{3}$ sample. The background approximated with a 2 -nd order polynomial is subtracted. The solid line gives a fit with Eq. (3) with $W=6500 \mathrm{~K}$.

note that we have taken $B<0$, which is unreasonable for a metal. Even if we take $B=0$, i.e. $\rho_{n}=$ const, the fitting above $T_{P}$ becomes considerably worse (see the broken line). Below we shall introduce a modification of the model above $T_{P}$ explaining the slower drop of the CDW fluctuations.

Let us now probe the model for the values which commonly characterize thermodynamic transitions, such as thermal expansion (TE), Young modulus $(Y)$ and specific heat $c_{p}$ (see e.g. [5]).

To perform TE measurements, we have developed an interferometric technique for measurements of needle-like samples $[19,20]$. Fig. 2 gives the temperature dependence of the relative length change $\delta l / l$ for $\mathrm{TaS}_{3}$ in the vicinity of the PT. To exclude the contribution of length hysteresis [19], we present the half-sum of the results obtained upon heating and cooling the sample. A similar curve results if we apply electric field exceeding $E_{T}$ to remove metastability each time before measuring $l$. Evidently, the curve presented is close to equilibrium. For all our measurements cooling below $T_{P}$ results in a drop of length by about $10^{-5}$. This result is quantitatively similar to that obtained for $\mathrm{K}_{0.3} \mathrm{MoO}_{3}$ in the in-chain direction [21].

TE at the PT has been discussed in [21]. E.g., it could be understood within the anharmonic model, taking into account the anharmonic effect of the lattice distortion associated with the CDW. Without deepening into details, we just assume that the length increase with heating is proportional to the fraction of the normal phase. Thus, the $l(T)$ step should be described by Eq. (3). The fit with $W=6500 \mathrm{~K}$ is quite nice (Fig. 2, the solid line).

As another example we consider the Young modulus temperature dependence, $Y(T)$. One can expect a drop of $Y$ due to the same anharmonic effects. So, with $T \rightarrow T_{P}$ from above one can expect a decrease of $Y$ proportional to the fraction of the CDW volume. However, 


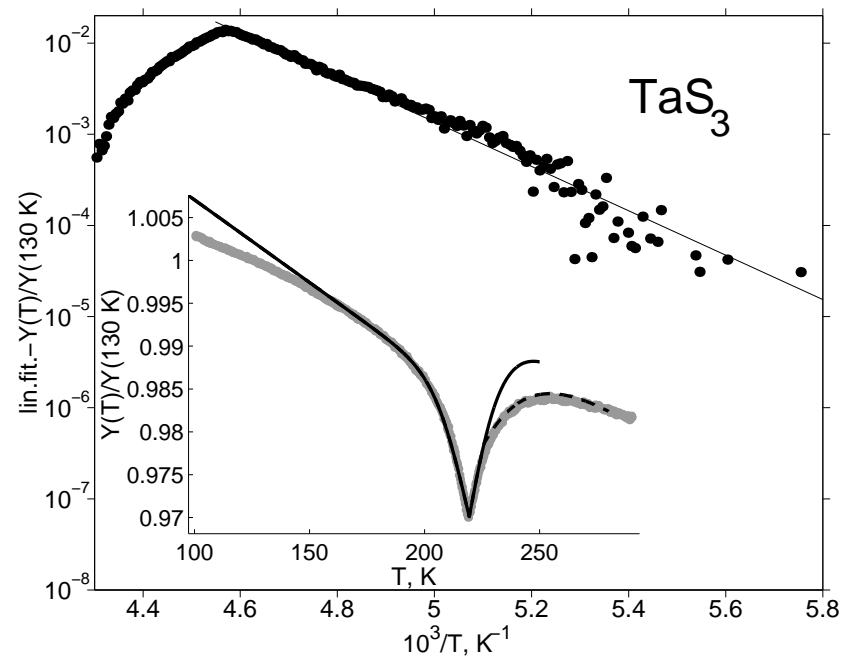

FIG. 3. Arrhenius plot of the deviation of $Y$ from a linear dependence $Y(T)$. The slope of the solid line corresponds to $W=5600 \mathrm{~K}$. The inset shows the fit of the whole $Y(T)$ curve. The broken line takes into account that for high $v\left(T>T_{P}\right)$ $1-v \propto \exp (-W / 2 T)$. The data are taken from [6].

this is not the whole effect.

Let us recall that depinning of the CDW below $T_{P}$ reduces the Young modulus $[4,22,23]$. (This effect is associated with fast relaxation of the CDW deformations which in the pinned state contribute to $Y$ [23].) As we mentioned above, with increasing $T$, the fluctuations result in the spontaneous depinning of the CDW $[14,17,18]$, the concentration of the depinned state growing as $\exp (-W / T)[14]$, which results in the drop of $Y$ with approaching $T_{P}$ from below. Thus, a dip of $Y(T)$ is expected at $T_{P}$ [24]. The value of the depinning drop of $Y$ depends on the particular compound. Being anomalously strong for $\mathrm{TaS}_{3}[22]$, it is not observed for the blue bronze, $\delta Y / Y<5 \cdot 10^{-5}[25,26]$. Thus, the dip in $Y(T)$ at $T \rightarrow T_{P}-0$ should be large for $\mathrm{TaS}_{3}$, and much weaker, if any, for the blue bronze. This expectation agrees with the experiment: inset to Fig. 3 shows $Y(T)$ for $\mathrm{TaS}_{3}$ from [6]. Large drop of $Y$ is seen with $T$ approaching $T_{P}$ both from above and from below, whereas only a small dip of $Y(T)$ at $T \rightarrow T_{P}$ from below is observed for the blue bronze in the in-chain direction $[4,5]$.

Fig. 3 shows the dependence $\delta Y(T)$ below $T_{P}$ in the Arrhenius axes (a linear dependence $Y(T)$ is subtracted). The slope of the solid line gives the activation energy $5600 \mathrm{~K}$, giving a good fit nearly up to $T_{P}$. To fit the whole $Y(T)$ curve we present $Y$ as $v Y_{n}+v_{p} Y_{p}+v_{r} Y_{r}$, where the indices $n, p$ and $r$ refer to the normal, pinned and relaxed states respectively $\left(v+v_{p}+v_{r}=1\right)$. The drop of $Y$ in comparison with the normal state can be presented as $\delta Y=\left(Y_{r}-Y_{n}\right) v_{r}+\left(Y_{p}-Y_{n}\right) v_{p}$, where $v_{r}=(1-v) \min \left(f_{r} \exp (-W / T), 1\right)$, and $v_{p}=\left(1-v-v_{r}\right)$. The inset to Fig. 3 shows the fit with $W=6000 \mathrm{~K}$, $f_{r}=7.8 \cdot 10^{11}, v_{0} \tau f_{a}=6 \cdot 10^{11},\left(Y_{r}-Y_{n}\right)=0.0443$,

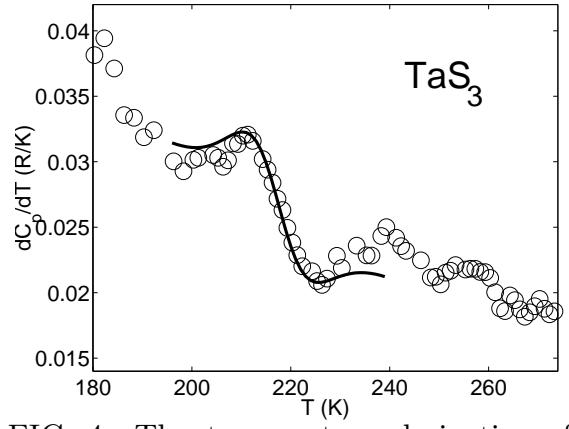

FIG. 4. The temperature derivative of the specific heat of $\mathrm{TaS}_{3}$. The fit is given by $\mathrm{d}^{2} v / \mathrm{d} T^{2}$ (Eq. (3)) with $W=10000 \mathrm{~K}$. The data are taken from [8].

$\left(Y_{r}-Y_{p}\right)=0.0111$ in the normalized units. The fit is quite nice, but above $T_{P}$ the fluctuations fall down slower than the fit gives, as it could be expected (recall also Fig. 1).

It is clear from the examples above that the PT consists in a gradual switching to the state as if having higher free energy, and thus looks as a smeared-out 1st order transition. The common check for the 1st order transition is the latent heat. Because of the smearing out one can expect a maximum of specific heat $c_{p}$. A cusp-like feature is clearly seen on the $c_{p}(T)$ curve for the blue bronze [5]. Recently a similar feature has been observed also for $\mathrm{TaS}_{3}$ [8]. Being very faint, it was detected as a zigzag pattern on the derivative $\mathrm{d} c_{p} / \mathrm{d} T$. Fig. 4 presents the data from [8] together with $\mathrm{d}^{2} v / \mathrm{d} T^{2}$, Eq. (3) (the background change of $c_{p}$ is approximated with a straight line). It is clear that the form of the feature is at least approximately described by our model. Other words, the CDW formation is accompanied by a smeared out step of latent heat whose width is of the order of $T_{P}^{2} / W$. Note that the values of the latent heat $Q \approx 0.25 R \cdot \mathrm{K}$ $=5 \cdot 10^{4} \mathrm{~J} / \mathrm{m}^{3}[8]$ ( $R$ is the universal gas constant) and the length change $\delta l / l=10^{-5}$ appear to be consistant with the Clausius-Clapeyron equation $\mathrm{d} T_{p} / \mathrm{d} \sigma=-T_{p} \frac{\delta l}{l} / Q$ if one takes $\mathrm{d} T p / \mathrm{d} \sigma \sim 1 \mathrm{~K} / \mathrm{kbar}[15,6]$ ( $\sigma$ is the stress along the chains) [27].

Above $T_{P}$ one should bear in mind the small sizes of the remnant CDW volumes $v_{c}$. As soon as they shrink down below $v_{0}$, Eq. (2) is no longer valid, because the new normal volume due to the LGS cannot exceed $v_{c}$. With simple assumptions at high enough $T$ one can obtain $(1-v) \propto \exp (-W / 2 T)$; the appropriate fit for $Y(T)$ is given with a broken line in the inset to Fig. 3. This consideration also explains the behavior of the $R(T)$ curve above $T_{P}$ (Fig. 1).

Thus, the LGS model fairly describes the temperature evolution of the principle parameters in the vicinity of the PT. All the fits proposed have transparent physical sense. The main parameter of the model, - the energy $W$, is close to the values for the barrier characterizing thermally initiated PS [12-14]. Evidently, LGS is gov- 
erned by the same process as PS. (Some indications of the connection between PS and the PT have been given in the early works $[10,11,14,28]$.) So, it would be natural to consider excitation of dislocation loops [29] as a precursor effect below $T_{P}$. Excitation of the dislocation loops is being considered as a possible origin of softening of solids [30] or similar transitions in liquid helium and HTSCs [31]. This approach gives critical expansion and proliferation of the loops due to their mutual screening. The apparent absence of the critical behavior in our case could mean that the CDW excitations practically do not interact up to $T_{P}$. Note that while for a conventional crystal the smallest possible radius of a dislocation loop is of the order of the lattice constant, for the CDW it is of the order of $\xi_{\perp}$. Such an object (i.e. an amplitude soliton) covers a volume $\sim \xi^{3} \equiv \xi_{\|} \xi_{\perp}^{2}$, where $\xi_{\|}$and $\xi_{\perp}$ are the in-chain and the transverse amplitude correlation lengths respectively; the soliton can perturb a still higher volume, where, say, the conductivity is increased. Thus, the condition $v=1 / 2$ can be achieved when the concentration of the excitations is still $\ll 1 / \lambda s$, where $\lambda$ is the CDW wavelength, and $s$ is the area per chain.

The concentration of the solitons could be estimated as $\frac{1}{\lambda s} \exp (-W / T)$, where $W$ is the energy of such an excitation. Then, $v \sim \frac{\xi^{3}}{\lambda s} \exp (-W / T)$. At $T=T_{P}$ we have $\frac{\xi^{3}}{\lambda^{3}} \exp (-W / T) \approx 1$, and come to the estimate:

$$
T_{P} \simeq \frac{W}{\ln \left(\xi^{3} / \lambda s\right)}
$$

With $\xi^{3} / \lambda s=10^{3}$ we obtain $T_{P}=W / 7$, which can give an idea of the low value of $T_{P}$ in comparison with $W$. A higher ratio $W / T_{P}$ might be obtained if we take into consideration the large wavelengths of the fluctuations of the CDW stress along the chains. According to [9] they can considerably exceed $\xi_{\|}$and, consequently, the LGS volumes could appear much larger than $\xi^{3}$.

According to our estimates, the excitations would begin to turn into dislocation loops, only above $T_{P}$. So, within the model, the metallic state develops at lower temperature than the critical behavior is expected to begin. As far as we comprehend, the approach proposes a new type of phase transition, which does not comprise $T_{P}$ as a parameter. At the same time, the PT in a sense resembles a 1st order transition. The model successfully works both below and above $T_{P}$, though further extrapolation of the approach to higher temperatures requires further development of the model. The underlying microscopic mechanisms of the LGS also needs deeper understanding. Though the model requires further grounds it gives a limpid insight into the processes inside the CDW near $T_{P}$.

We are thankful to J.W. Brill, G. Mozurkewich and D. Starešinić for granting the experimental results at our disposal, to V.V. Frolov and R.E. Thorne for furnishing the samples, to V.I. Anisimkin, L. Burakovsky and
S.N. Artemenko for helpful discussions. This work has been supported by the RFBR (grants No 01-02-17771, 0202-17301), Jumelage (CNRS and RFBR), INTAS (grant No 01-0474) and by the State programme "Physics of Solid-State nanostructures".

[1] M.E.Itkis, F.Ya.Nad' Pis'ma Zh. Eksp. Teor. Fiz. 39, 373 (1984) [JETP Lett 39, 448 (1984)].

[2] B. P. Gorshunov et al., Phys. Rev. Lett., 73, 308 (1994).

[3] V. Ya. Pokrovskii, S. V. Zaitsev-Zotov, P. Monceau, Phys. Rev. B 51, R13377 (1997)

[4] J. W. Brill, in Handbook of Elastic Properties of Solids, Liquids, and Gases, edited by M. Levy, H. E. Bass, and R. R. Stern (Academic Press, New York, 2001), Vol. II, pp. 143-162.

[5] J.W. Brill et al., Phys. Rev. Lett 74, 1182 (1995).

[6] G. Mozurkewich, R.L. Jacobsen, Synth. Met. 60, 137 (1993).

[7] P. Ehrenfest, Leiden Comm. Suppl. 75b, 8 (1933); L.R. Testardi, Phys. Rev. B 3, 95 (1971); ibid 12, 3849 (1975).

[8] D. Starešinić et al., Eur.Phys.J.B 29, 71 (2002).

[9] S.N. Artemenko, J.Phys.IV 12, Pr9-77 (2002); private communication.

[10] V.Ya. Pokrovskii and S.V. Zaitsev-Zotov, Europhys.Lett., 13, 361 (1990).

[11] V.Ya. Pokrovskii, S.V. Zaitsev-Zotov, Synth. Met, 4143, 3899 (1991)

[12] J.C. Gill, J.Phys.C: Solid State Phys., 19, 6589 (1986).

[13] D.V. Borodin, S.V. Zaitsev-Zotov and F.Ya. Nad', Zh. Eksp. Teor. Fiz. 90, 618 (1986); [Sov Phys. JETP 63, 194 (1986)].

[14] V. Ya. Pokrovskii and S. V. Zaitsev-Zotov, Phys. Rev. B 61, 13261 (2000)

[15] P.Monceau in: Electronic Properties of Inorganic Quasione-dimensional Conductors, Part 2. Ed. by P. Monceau. Dortrecht: D.Reidel Publ. Comp., 1985.

[16] If instead of resistivities we sum up conductivities (connection in parallel), the result is similar. Actually, one can expect a weak criticality associated with the current percolation through the normal-phase cluster, but this concerns only the transport properties.

[17] J.C. Gill, Synth. Met. 43, 3917 (1991).

[18] J. McCarten et al., Phys. Rev. B 46, 4456 (1992).

[19] A. V. Golovnya, V. Ya. Pokrovskii, and P. M. Shadrin, Phys. Rev. Lett. 88, 246401 (2002).

[20] A.V. Golovnya, V.Ya. Pokrovskii, Rev. Sci. Instrum. 74, 4418 (2003).

[21] M.R. Hauser, B.B. Plapp, and G. Mozurkevich, Phys. Rev. B 43, 8105 (1991).

[22] R.L. Jacobsen and G. Mozurkewich, Phys. Rev. B 42, 2778 (1990).

[23] G. Mozurkewich, Phys. Rev. B 42, 11183 (1990).

[24] Note, that the treatment of the $Y(T)$ anomaly proposed in [K. Maki, Phys. Rev. B. 41, 2657 (1990).] also distin- 
guishes pinned and depinned CDW.

[25] L.C. Bourne, and A. Zettl, Solid State Commun. 60, 789 (1986).

[26] To our understanding [19], the effect is due to 3D features of the electronic structure, which are individual for each compound.

[27] J.W. Brill, 2003, private communication.

[28] S.V. Zaitsev-Zotov, V.Ya. Pokrovskii, Abstracts of the XXV All-Union Session on the Low-Temperature Physics, Leningrad, 1988, part 3, p. 112 (in Russian).

[29] K. Maki, Physica 143B, 59 (1986). S. Ramakrishna et al., Phys. Rev. Lett. 68, 2066 (1991).

[30] S. Panyukov and Y. Rabin, Phys. Rev. B 59, 13657 (1999).

[31] G.A. Williams, Phys. Rev. Lett. 82, 1202 (1999). 